\title{
XMM-Newton Detection of Extended X-ray Emission from the Saturn Nebula
}

\author{
Martín A. Guerrero, Robert A. Gruendl, \& You-Hua Chu \\ Department of Astronomy, University of Illinois at Urbana-Champaign, \\ 1002 W. Green St., Urbana, IL 61801, USA
}

\begin{abstract}
XMM-Newton EPIC observations of the planetary nebula NGC 7009, the Saturn Nebula, have detected extended X-ray emission from its central cavity. The diffuse X-ray emission must originate in the shocked fast stellar wind. Spectral analyses show that the temperature of the hot gas is $1.7 \times 10^{6} \mathrm{~K}$. The RMS density derived from the volume emission measure is a few tens $\mathrm{H}$-atom $\mathrm{cm}^{-3}$. The hot gas does not appear over-pressurized with respect to the nebular shell. The Saturn Nebula may represent an evolutionary stage at which the dynamic effects of the hot gas in the central cavity on the cold nebular shell starts to decline due to the diminishing strength of the fast stellar wind and the expansion of the central cavity.
\end{abstract}

\section{Introduction}

It is commonly accepted that the shell structure of a planetary nebula $(\mathrm{PN})$ is formed by the interaction of the current fast wind of the central star and the previous slow AGB wind. In this interacting-stellar-winds model, the central cavity of the $\mathrm{PN}$ is expected to be filled with shocked fast wind at temperatures $10^{6}-10^{8} \mathrm{~K}$ that should be detectable in X-rays.

The Saturn Nebula (NGC 7009) has a bright, $25^{\prime \prime} \times 10^{\prime \prime}$ inner shell surrounded by a $25^{\prime \prime} \times 20^{\prime \prime}$ envelope, with two ansae extending out to $25^{\prime \prime}$ from the central star. The sharp-edged morphology of the inner shell suggests that it is the compressed AGB wind swept-up by the fast stellar wind. A $30 \mathrm{ks}$ $X M M-N e w t o n$ EPIC observation of the Saturn Nebula has detected extended $\mathrm{X}$-ray emission. These observations have been used to investigate the spatial distribution and the physical conditions of the X-ray-emitting gas.

\section{Spatial and Spectral Analysis}

The X-ray emission from the Saturn Nebula is clearly extended. Its spatial distribution in the EPIC image can be described by an elliptical Gaussian with FWHM $\sim 23^{\prime \prime} \times 14^{\prime \prime}$ (Fig. 1-left). The excellent correspondence between the $\mathrm{X}$-ray emission and the nebular shell of the Saturn Nebula suggests that the hot, X-ray-emitting gas is confined within the central cavity.

The background-subtracted EPIC/MOS and EPIC/pn spectra of the Saturn Nebula show spectral features consistent with thermal plasma emission, for instance, the peak at $\sim 0.55 \mathrm{keV}$ corresponds to the He-like triplet at $\sim 0.57$ $\mathrm{keV}$ (Fig. 1-right). We have therefore adopted a thin plasma emission model 



Figure 1. (left) PSF-deconvolved EPIC image overplotted on an $H S T$ WFPC2 $\mathrm{H} \alpha$ image of the Saturn Nebula. The contour levels are chosen at $25,50,75$, and $90 \%$ of the emission peak. (right) $X M M$ Newton EPIC/pn, EPIC/MOS1, and EPIC/MOS2 spectra of the Saturn Nebula. The spectra are overplotted with the best-fit Raymond \& Smith model (1977) to the EPIC/pn and the combined EPIC/MOS spectra.

(Raymond \& Smith 1977) with nebular abundances (Kwitter \& Henry 1998) in order to fit the observed EPIC spectra and determine the physical conditions of the X-ray-emitting gas. The best-fit model has a plasma temperature of $T=$ $1.7 \pm 0.110^{6} \mathrm{~K}$ (or $k T=0.147 \mathrm{keV}$ ), and an absorption column density of $N_{\mathrm{H}}$ $=6.0_{-2.5}^{+3.0} 10^{20} \mathrm{~cm}^{-2}$, a volume emission measure of $1.9_{-0.4}^{+0.5} 10^{54} d^{2} \mathrm{~cm}^{-3}$ where $d$ is the distance to the Saturn Nebula in kpc.

At a distance of $1.2 \mathrm{kpc}$, the volume emission measure corresponds to a density of a few tens $\mathrm{H}$-atom $\mathrm{cm}^{-3}$. The thermal pressure of the hot gas is comparable to that of the nebular shell. The observed (absorbed) X-ray flux in the $0.2-2.5 \mathrm{keV}$ energy range is $1.2 \times 10^{-13} \mathrm{ergs} \mathrm{cm}^{-2} \mathrm{~s}^{-1}$, and the unabsorbed (intrinsic) X-ray flux is $3.6 \times 10^{-13} \mathrm{ergs} \mathrm{cm}^{-2} \mathrm{~s}^{-1}$. At $1.2 \mathrm{kpc}$, this corresponds to $L_{\mathrm{X}}=6 \times 10^{31} \mathrm{ergs} \mathrm{s}^{-1}$.

\section{Results}

XMM-Newton EPIC observations of the Saturn Nebula have shown that the diffuse X-ray emission is confined within the central cavity. The hot gas in this cavity is not over-pressurized with respect to the nebular shell. The X-ray luminosity, density, and pressure of the hot gas in the Saturn Nebula are the lowest among the four PNe whose diffuse X-ray emission has been detected. These differences may be associated with nebular evolution, and suggest that the pressure-driven phase of a PN is short, lasting for only $\sim 1,000 \mathrm{yr}$.

\section{References}

Raymond, J. C., \& Smith, B. W. 1977, ApJS, 35, 419

Kwitter, K. B., \& Henry, R. B. C. 1998, ApJ, 493, 247 